\title{
Superconducting superstructure for the TESLA collider: A concept
}

\author{
J. Sekutowicz \\ DESY, Notkestrasse 85, Hamburg 22603, Germany \\ M. Ferrario \\ INFN, Via Enrico Fermi 40, Frascati 00044, Italy \\ Ch. Tang \\ Tsinghua University, Beijing 100084, China \\ (Received 8 September 1998; published 8 June 1999)
}

\begin{abstract}
We discuss a new layout of a cavity chain (called superstructure) allowing, we hope, a significant cost reduction due to a simplification of the rf system of the TESLA linear collider. The proposed scheme increases the fill factor and thus makes an effective gradient of the accelerator higher. In this paper computations and preliminary measurements on existing copper models of the TESLA Test Facility accelerating structures are presented. A new copper model of the scheme has been ordered, made of four 7-cell standing wave cavities, which according to the results of computations and measurements seems to be the most promising version. Experiments with a beam will be necessary to prove that the proposed layout can be used for the acceleration. [S1098-4402(99)00039-7]
\end{abstract}

PACS numbers: 84.90.+a, 41.20.-q, 41.75.Ht

\section{INTRODUCTION}

Recent tests have shown that two main technical specifications for the TESLA superconducting cavitiesan accelerating gradient $E_{\text {acc }}$ of $25 \mathrm{MV} / \mathrm{m}$ and a quality factor $Q_{0} \geq 5 \times 10^{9}$ - are achievable for $1300 \mathrm{MHz}$ bulk niobium cavities $[1,2]$. The R\&D program at DESY started in 1992 to develop superconducting technology for the future linear collider is being continued in order to obtain specifications reproducibly and to lower the cost of the technology.

The essential part of the total investment is the cost of the rf, meant here as the sum of the cost of accelerating structures with auxiliaries and the cost of the rf power distribution system. To reduce this cost more effort should be made to decrease the number of rf components, e.g., fundamental mode (FM) couplers, higher order mode (HOM) couplers, waveguides, circulators, and waveguide transformers, and to increase the effective gradient $E_{\text {eff }}$ in the collider.

In the present TESLA Test Facility (TTF) design there is $1 \mathrm{FM}$ coupler and $2 \mathrm{HOM}$ couplers per 9-cell cavity which is $1 \mathrm{~m}$ long. The dense positioning of FM couplers makes the rf power distribution system and cryostats more complex and thus more expensive. The effective accelerating gradient in both linacs will be $17.8 \mathrm{MV} / \mathrm{m}$ only, while the cavities will be operated at $25 \mathrm{MV} / \mathrm{m}$. There are two reasons for this: a small fill factor and an unflatness of the accelerating field. The fill factor, defined as the ratio fill factor $=$ (cavity active length) $/$ (cavity total length), has a low value of 0.75 . This results mainly from the $3 \lambda / 2$ long interconnections between cavities (see Fig. 1). This length was chosen at the beginning of the linear col- lider studies only to obtain good cavity separation for the accelerating mode and simplicity in the phase adjustment. The first argument will be discussed later, but $7 \mathrm{~km}$ of passive length appears too generous. The second argument is no longer valid since a variable coupler or a 3 -stub waveguide transformer, similar to those installed in the HERA $e^{-}$ring [3], can be applied in the rf input line of each cavity, to adjust the phase and the value of $Q_{\text {ext }}$, for reflection-free operation.

The cell-to-cell amplitude variation of the accelerating field is often $\sim 10 \%$. This leads to $\sim 5 \%$ reduction of $E_{\text {eff }}$ because the achievable gradient is usually limited by the cell with maximum amplitude. For the accelerating $\pi$ mode, the error of the field amplitude $\Delta A_{\text {cell }}$ in an individual cell is related to the frequency error $\Delta f_{\text {cell }}$ of the cell as follows [4]:

$$
\Delta A_{\text {cell }} \sim(N)^{2} \Delta f_{\text {cell }} / k_{c c},
$$

where $N$ is the number of cells in the cavity and $k_{c c}$ is the cell-to-cell coupling. Experience shows that TTF cavities with $N=9$ and $k_{c c}=1.9 \%$ are at the limit. Each mechanical, chemical, or thermal treatment perturbs the

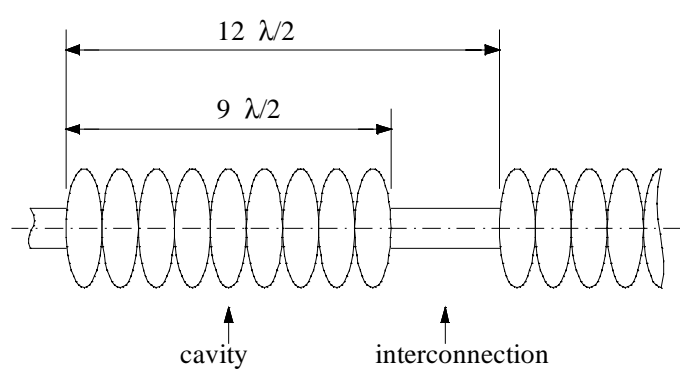

FIG. 1. The 9-cell cavity and the interconnection. 
field profile and the specification of field unflatness of less than $5 \%$ is hard to obtain. A significant cost reduction could be gained when $N$ increases due to the lower number of rf auxiliaries per unit length. Unfortunately, there are two fundamental limitations on $N$. First, the field profile, which is already a problem, becomes less stable proportional to $N^{2}$, as can be seen from Eq. (1). Second, the probability of trapping parasitic resonances within the structure is higher. This is especially dangerous for superconducting cavities because parasitic modes can have a big beam impedance due to high quality factor.

\section{SUPERSTRUCTURE}

To overcome limitations on $N$ and simultaneously to make interconnections shorter one may use the layout (superstructure) shown in Fig. 2. The idea is to couple cavities by short beam tubes to enable an energy flow from cavity to cavity instead of separating them by a long interconnection. In this scheme, similar to the present design, HOM couplers are attached to interconnections and each cavity (subunit) is equipped with a tuner. This layout increases the number of cells fed by one FM coupler, avoiding the two limitations we mentioned above. Both the field flatness and the HOM damping can still be handled at the subunit level. The number of modes in the FM passband is $N N_{s}$, where $N_{s}$ is a number of coupled cavities. The cavity-to-cavity coupling, $k_{s s}$, causes each of the $N$ modes of a single cavity to split into a group of $N_{s}$ resonances. The synchronism between the ultrarelativistic beam and the accelerating field requires that the shortest possible interconnection be $\lambda / 2$ long. When $N$ is an odd number, the $\pi-0$ mode ( $\pi$ is cell-to-cell phase advance and 0 is cavity-to-cavity phase advance) can be used for the acceleration. The accelerating field profile of this mode in two neighboring cavities is shown in Fig. 3. When the length is fixed, coupling $k_{s s}$ depends on the diameter of the interconnecting tube and on the field profile. It varies from mode group to mode group. For the practicable diameter of the interconnection $k_{s s}$ is smaller than $k_{c c}$. Figure 4 shows an example of $k_{s s}$ for different groups of a superstructure based on a 9-cell cavity with end beam tubes of $110 \mathrm{~mm}$ diameter. In this diagram, mode groups are

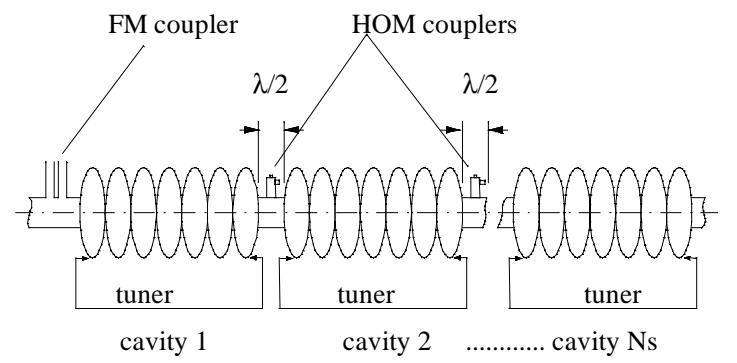

FIG. 2. An example of $N_{s}$ 7-cell cavities coupled by short interconnections (superstructure).

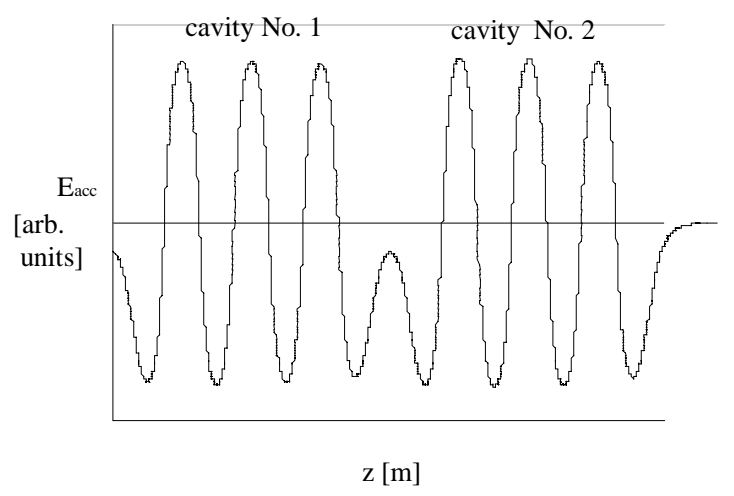

FIG. 3. An example of $E_{\text {acc }}$ vs $z$ in two neighboring 7-cell cavities excited in the $\pi-0$ mode.

represented by the cell-to-cell phase advance. Although $k_{s s}$ is small, with the help of tuners one can correct the frequency of each subunit to equalize the mean value of the field amplitude in all subunits (not in the cells within one subunit). Additionally one should note that even $k_{s s}$ is small; perturbation of the cavities phasing resulting from the beam loading will be negligible. This is mainly due to the high value of the loaded $Q\left(>10^{6}\right)$ for the TESLA superconducting structures. According to Eq. (82) in [5] for a few coupled cavities, this phase shift will be less than $1^{\circ}$.

\section{SUPERSTRUCTURE BASED ON THE 7-CELL SUBUNIT}

The performance of the superstructure depends on the field profile stability within each subunit. There are two ways to make the accelerating field more stable: increasing $k_{c c}$ and reducing $N$ in the subunit; see Eq. (1). The first proposed superstructure [6], made of four 9-cell cavities, had a more stable field due to the enlarged midiris diameter and thus 3 times stronger $k_{c c}$ than the TTF cavity. Unfortunately, the bigger aperture has some disadvantages as compared to the present design: higher $E_{\text {peak }} / E_{\text {acc }}$ and $H_{\text {peak }} / \mathrm{E}_{\text {acc }}$, and lower impedance $(R / Q)$. These caused the total improvement in $E_{\text {eff }}$ to be rather small. Nevertheless, the large iris is better for alternative fabrication methods such as hydroforming or spinning. These

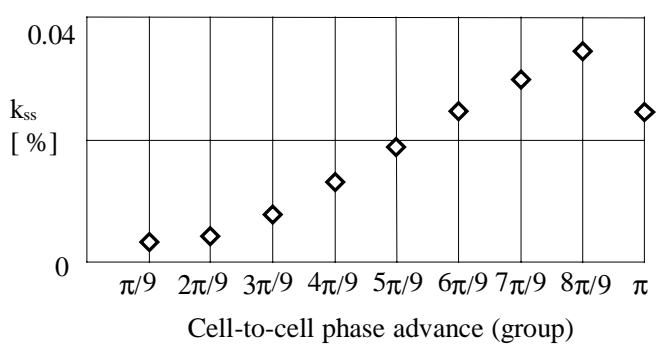

FIG. 4. $k_{s s}$ for different mode groups in the superstructure based on a 9-cell cavity with a $110 \mathrm{~mm}$ beam tube. 
methods are under development, and may in the future significantly reduce the cavity cost $[7,8]$. In addition, much lower transversal and longitudinal loss factors make this superstructure suitable for the acceleration of bunches with high population of particles, as in the case of the muon collider.

Today, the most probable future scenario for the energy upgrade of the TESLA collider, above $500 \mathrm{GeV}$, is operation at higher accelerating gradient and/or making the collider longer [9]. This brought us to an alternative version of the superstructure [10], based on the TTF shape with modified end cells and reduced $N$ from 9 to 7 to improve field stabilization according to (1). As before, the superstructure is made of four subunits. This version keeps $E_{\text {peak }} / E_{\text {acc }}$ and $H_{\text {peak }} / E_{\text {acc }}$ low, as in the TTF cavity, and makes operation above $25 \mathrm{MV} / \mathrm{m}$ more feasible, since the maximum electric and magnetic fields on the $\mathrm{Nb}$ wall are further from the theoretical limitations. Table I contains a list of parameters of the superstructure and the TTF cavity. The last row of the table shows $E_{\text {eff }}$ for the operation at $25 \mathrm{MV} / \mathrm{m}$. In the case of the superstructure, $E_{\text {eff }}$ is computed according to the bigger fill factor given in the third row and corrected with the expected field unflatness. This is scaled from the value observed for the TTF cavities proportional to the field instability factor shown in the fourth row. The resulting $E_{\text {eff }}$ value for the superstructure is higher than $E_{\text {eff }}$ of the TTF cavity by $19 \%$.

\section{A. Refilling of cells and bunch-to-bunch energy spread}

The most critical part of the numerical simulation is the calculation of the transient state and the bunch-to-bunch energy spread. Two codes, HOMDYN [11] and MAFIA $[12,13]$, showed that there is enough time to refill the cell's energy in the superstructure before the next bunch arrives. This result is not obvious since the coupling between subunits is very small. The computed energy gain for the proposed superstructure, when it is operated at $25 \mathrm{MV} / \mathrm{m}$, is shown in Fig. 5(a). The energy spread

TABLE I. Parameters of the superstructure and the TTF cavity.

\begin{tabular}{lcc}
\hline \hline \multicolumn{1}{c}{ Parameter } & Superstructure & TTF cavity \\
\hline Radius of mid/end iris $(\mathrm{mm})$ & $35 / 57$ & $35 / 39$ \\
$N / N_{s}$ & $7 / 4$ & $9 / 1$ \\
Fill factor & 0.875 & 0.75 \\
Field instability factor & & \\
$\quad N^{2} / k_{c c}\left(10^{3}\right)$ & 2.6 & 4.3 \\
$k_{c c}(\%)$ & 1.9 & 1.9 \\
$k_{s s} \pi$ group $(\%)$ & $3.6 \times 10^{-2}$ & $\ldots$ \\
$(R / Q)$ subunit/cavity $(\Omega)$ & 732 & 1030 \\
$E_{\text {peak }} / E_{\text {acc }}$ & 2.0 & 2.0 \\
$H_{\text {peak }} / E_{\text {acc }}[\mathrm{Oe} /(\mathrm{MV} / \mathrm{m})]$ & 41.8 & 41.8 \\
$E_{\text {eff }}(\mathrm{MV} / \mathrm{m})$ & 21.2 & 17.8 \\
\hline \hline
\end{tabular}
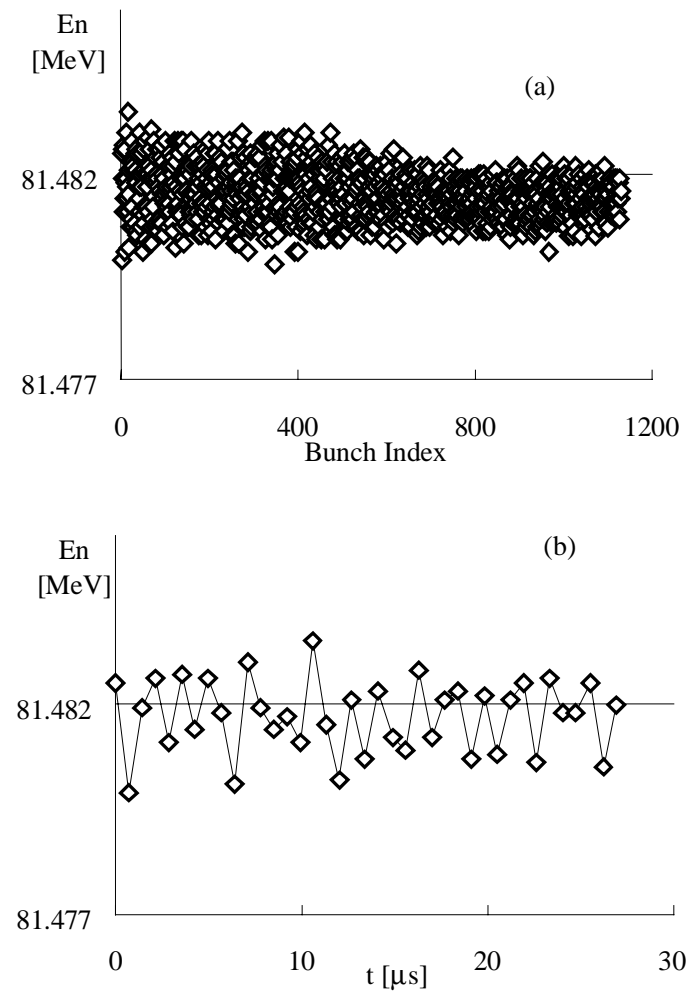

FIG. 5. (a) Energy gain for 1130 bunches accelerated with the superstructure. (b) Energy gain vs time for the first 40 bunches.

for the whole train of 1130 bunches is $<5 \times 10^{-5}$. It results from the interference of the accelerating mode with other modes from the $\pi$ group and the $6 \pi / 7$ group. The difference in energy is bigger at the beginning of the train [Fig. 5(b)] and becomes smaller at the end of the pulse due to the decay of interfering modes.

\section{B. The HOM damping scheme}

For the chosen diameter of the interconnecting tubes almost all HOMs are above cutoff. According to the computation, their field strength is high enough for damping with HOM couplers attached at the middle of the interconnections. In that way, each HOM coupler can be exploited to damp modes from two neighboring cavities. Such a scheme will require fewer HOM couplers than the current one, but this must be proven experimentally. The couplers have to couple both electrically and magnetically, due to a standing wave pattern of HOMs in interconnections. One should mention that the HOM couplers used at present already have this coupling characteristic providing the required $Q$ values in the range of $10^{4}-10^{5}$.

\section{Changes in the rf system}

In addition to the improvement in the effective accelerating gradient, the number of FM and HOM couplers 
TABLE II. Changes in the rf system.

\begin{tabular}{lcc}
\hline \hline & TTF design & Superstructure \\
\hline Number of FM couplers & 19230 & 6181 \\
Number of HOM couplers & 38460 & 24724 \\
Number of tuners and vessels & 19230 & 24724 \\
Power/FM coupler $(\mathrm{kW})$ & 208 & 640 \\
\hline \hline
\end{tabular}

will be significantly reduced, if the proposed scheme can be used for the acceleration. Table II shows the total amount of couplers, helium vessels, and tuners needed in the TESLA collider in two cases: when the layout of linacs is based on the current TTF cavity design and, alternatively, when it is based on the superstructure. The number of FM couplers is reduced by a factor of 3. This has major consequences for the cost of the whole rf system. The layout based on the proposed superstructure needs an additional $22 \%$ more tuners and liquid helium vessels due to 7 instead of 9 cells in a subunit. Nevertheless, the simplification in the rf system and the cryostat construction will dominate and a total cost reduction can be expected.

The power transferred by the FM coupler feeding the superstructure is $640 \mathrm{~kW}$. The new version of the FM coupler developed at DESY has been tested up to $1.6 \mathrm{MW}$ for the whole TESLA pulse length [14]. The limitation was due to the rf power source. Since this version already exceeds the power needed for operation of the superstructure at $25 \mathrm{MV} / \mathrm{m}$ we do not expect fundamental difficulties here.

\section{PRELIMINARY MEASUREMENTS}

Four existing copper models of 9-cell TTF cavities have been used for preliminary rf measurements on the superstructure. The length of beam tubes, the diameter of which is only $78 \mathrm{~mm}$, was adjusted to get a $k_{s s}$ value for the $\pi$ group equal to the value from Table I.

\section{A. Tuning of the FM field profile}

Each of the four cavities has been tuned individually for a field flatness of better than $98 \%$ and the chosen frequency of the $\pi-0$ mode. The operating cavities' boundary conditions are imposed by the nearby cavities or the beam pipe. We reproduced the operating boundary conditions for the tuning procedure by means of metallic plates $\lambda / 4$ apart from the last iris, for inner cavities' tuning, and a beam tube connected to the external iris for the outer cavities' tuning. After they had been connected in one superstructure, the field measured with an exciting antenna placed in the first cell and a pickup antenna in the last cell, as in the standard bead pull measurement, showed unflatness of $20 \%$. This was mainly due to the overlapping of neighboring modes with the $\pi-0$ mode (see Fig. 6) and some mechanical deformation of

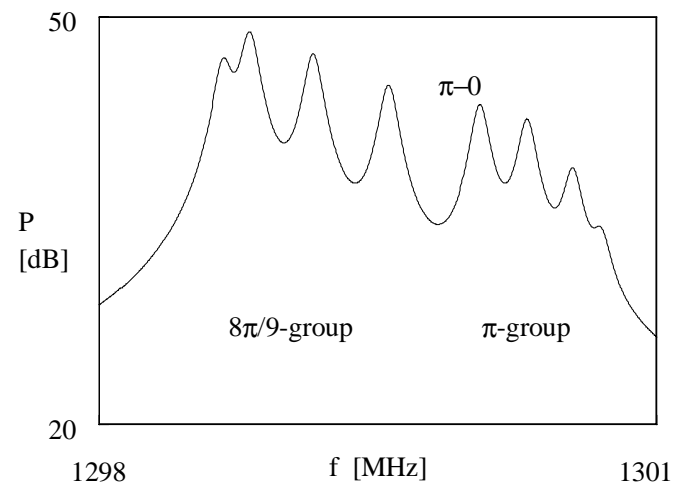

FIG. 6. Modes from the $8 \pi / 9$ group and the $\pi$ group when $Q_{0}=2.5 \times 10^{4}$.

end cells during the assembly. The influence of the overlapping has been simulated with the help of a lumped element circuits code. The computation has been done for $36 L C R$ resonators, coupled in four groups. The group-to-group coupling and the coupling within each group were equal to the values of $k_{s s}$ and $k_{c c}$ from Table I. Both couplings had capacitive character as it is common for cavities of this shape. The results are shown in Fig. 7. The whole chain has been tuned for the unloaded superconducting case, $Q_{0}=3 \times 10^{10}$. The achieved field flatness for all 36 amplitudes was better than $99 \%$. In the next computation, resistors in the whole chain were changed to simulate superconducting cells loaded with the beam $\left(Q=3.4 \times 10^{6}\right)$. The computed field profile stayed unchanged. Further, the resistors were modified a second time to simulate the copper model of the superstructure with $Q_{0}=2.5 \times 10^{4}$. This time the field profile was perturbed significantly and it was very similar to one that we have measured with the bead-pull technique for these antenna positions. To suppress both nearest modes the antennae were placed at the midcells in which these modes have a small field amplitude. The measured field profile of $\pi-0$ mode for these antenna positions is shown in Fig. 8. The unflatness $<4 \%$ of the field was obtained after small frequency correction of the

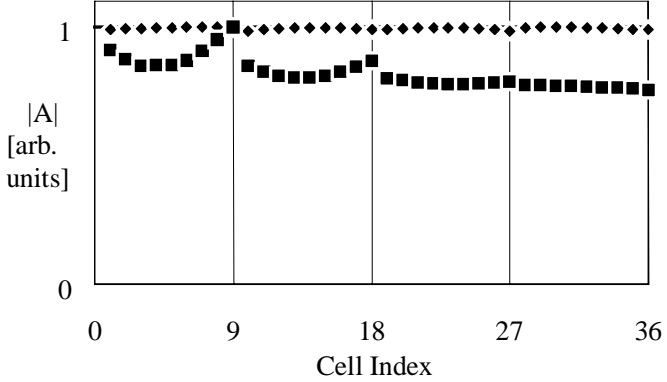

FIG. 7. Computed amplitudes in an $L C R$ replacement circuit of four 9-cell TTF cavities: $(\square) Q_{0}=2.5 \times 10^{4},(\diamond) Q_{0}=$ $3 \times 10^{10}$ and $Q=3.4 \times 10^{6}$. 


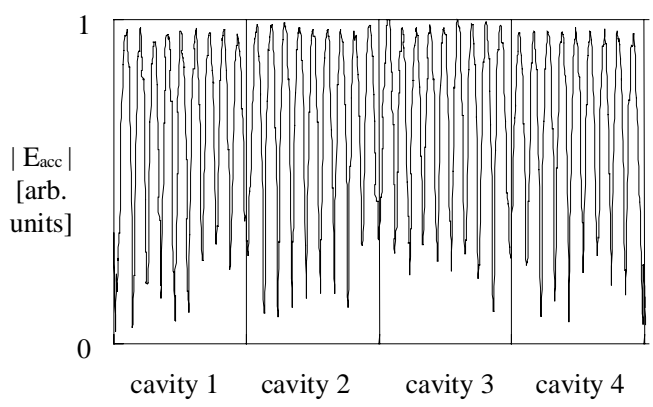

FIG. 8. The measured field profile in the preprototype of the superstructure.

end cells. Unfortunately, in $\mathrm{Nb}$ cavities no openings are available in the inner cells for antenna attachment. This makes the control of the field profile at room temperature more complicated and allows no mechanical perturbation of well pretuned subunits during the assembly of the superstructure. The stability of the FM field pattern was tested for an inner and an outer subunit. In both cases, measurement began with the tuned field profile shown above. For a detuning of a few $\mathrm{kHz}$ the perturbation $\Delta A / A$ of the mean field amplitude of a subunit was proportional to the subunit frequency perturbation $\Delta f$,

$$
\Delta A / A=-0.01 / \mathrm{kHz} \Delta f .
$$

The measurement has proven that the sensitivity of the field pattern is not critical. The tuners and the frequency control system applied in the TTF linac already allow adjustment of the frequency of an individual cavity with an accuracy of $0.8 \mathrm{~Hz}$.

On the other hand, when necessary during the linac operation by a proper detuning one can lower the accelerating field of a subunit showing degradation in performance, while simultaneously keeping the field of other subunits at the former level.

\section{B. Transient state of the fundamental mode}

The transient state of each cell from the chain has been computed with the help of the HOMDYN code [15]. For cells No. 9 and No. 36 the computed curves are compared with the measured curves in Figs. 9(a) and 9(b).

The agreement between the computed and measured results is good. Small differences come mainly from a variation of the cell-to-cell coupling in the copper models. The variation of $k_{c c}$ causes some additional effect for other modes from the FM passband. This is discussed in the following subsection.

\section{Other modes from the FM passband}

Due to manufacturing errors in the iris region, $k_{c c}$ varies from cell to cell and from subunit to subunit. As a result when the $\pi$ mode frequency of all subunits is equal, the frequencies of other modes from the FM
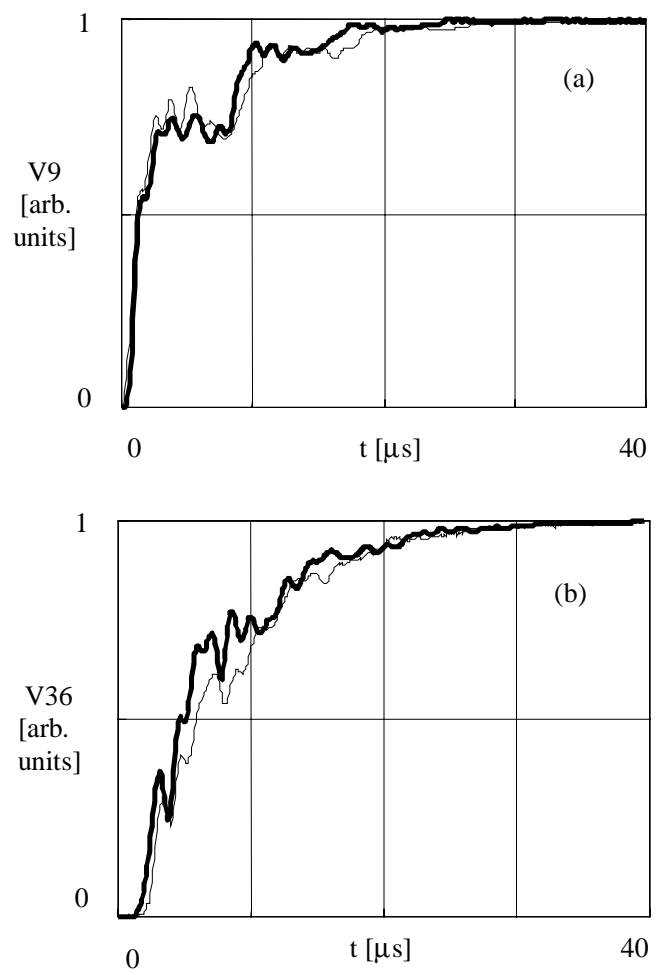

FIG. 9. (a) Transient in cell No. 9: computed (bold line), measured (standard line). (b) Transient in cell No. 36: computed (bold line), measured (standard line).

passband differ from each other. As an example, the deviation from mean values of the four lowest modes for existing copper models is shown in Fig. 10. When identical subunits are assembled in the superstructure these modes form groups with small $k_{s s}$ coupling (see Fig. 4). In a real case, the uncorrected difference in frequency causes the subunits to stay uncoupled for these groups and behave like separate cavities. The $(R / Q)$ values of these resonances are small since a cancellation of impedance takes place within each subunit, as it does for the uncoupled TTF cavities. The computed $(R / Q)$ values for the 28 passband modes of superstructure are

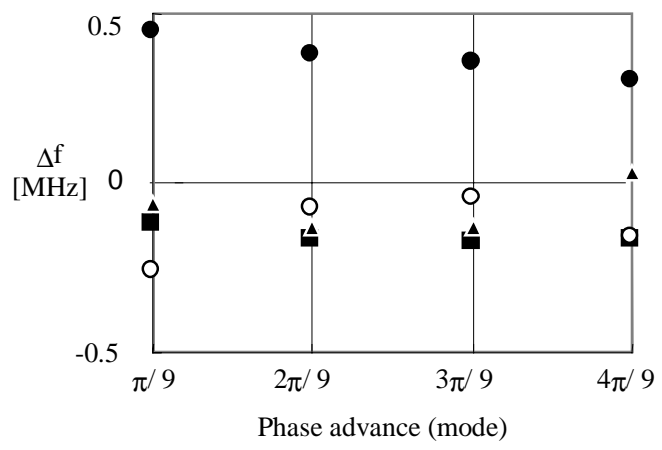

FIG. 10. Frequency deviation from the mean values for $\mathrm{Cu}$ models of a 9-cell TTF cavity: cavity No. 1 (O), cavity No. 2 $(\boldsymbol{\square})$, cavity No. $3(\bigcirc)$, and cavity No. $4(\mathbf{\Delta})$. 


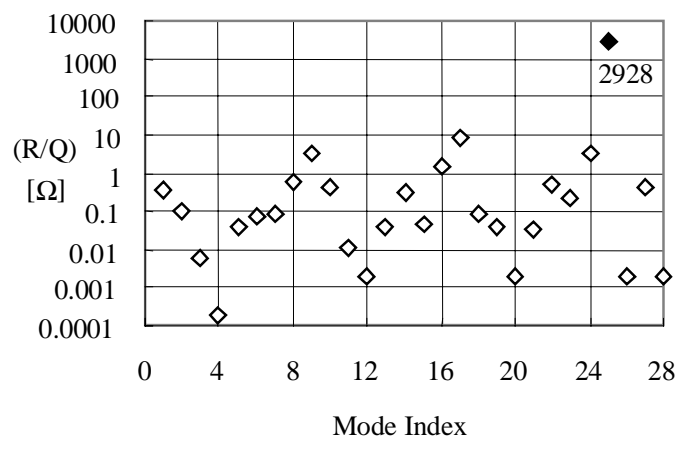

FIG. 11. $(R / Q)$ 's for modes from the FM passband: $(\diamond)$ accelerating mode, $(\diamond)$ other modes.

shown in Fig. 11. An interaction between the beam and these resonances has been estimated with the help of the HOMDYN code. For the computation, the frequency of a mode with the highest $(R / Q)$ in each group has been shifted to the frequency of the nearest harmonic to simulate the worst case, i.e., a synchronous excitation of the mode. The $Q$ values were chosen in the following way: for 16 modes from the $\pi / 7,2 \pi / 7,3 \pi / 7$, and $4 \pi / 7$ groups (small $k_{s s}$ ), $Q$ was set to $10^{9}$ with an assumption of no coupling to the input via the FM coupler (no damping). For the other 11 modes from the $5 \pi / 7,6 \pi / 7$, and the $\pi$ groups, $Q$ was scaled from the loaded $Q$ of the accelerating mode, according to their field pattern in the superstructure. These $Q$ values are shown in Fig. 12. The computed energy spread is of the order of $6 \times 10^{-3}$ (Fig. 13, upper curve). It exceeds the specification but can be significantly reduced, to the level of $10^{-3}$, when the injection time is properly chosen in order to balance the beam induced voltage by the rising generator voltage (Fig. 13, lower curve). Four modes, No. 9, No. 16, No. 17, and No. 24, give a 95\% contribution to the computed energy spread. The original frequencies of these modes were shifted for the computation by $0.61,0.52,0.32$, and $-0.88 \mathrm{MHz}$, respectively. Since the energy spread for the original spectrum was smaller than $5 \times 10^{-5}$, the most effective

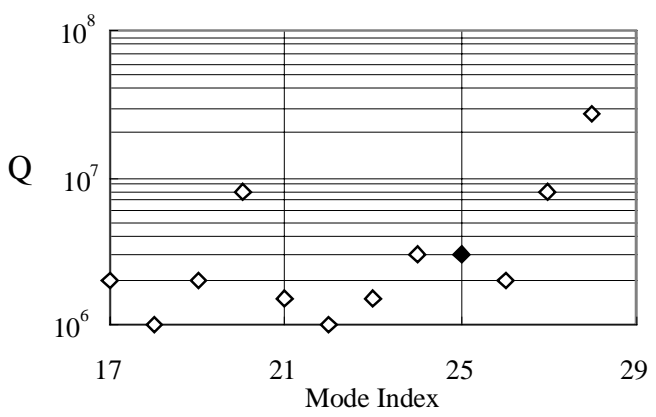

FIG. 12. The computed quality factors for modes from the $5 \pi / 7,6 \pi / 7$, and $\pi$ groups: $(\diamond)$ accelerating mode, $(\diamond)$ other modes.

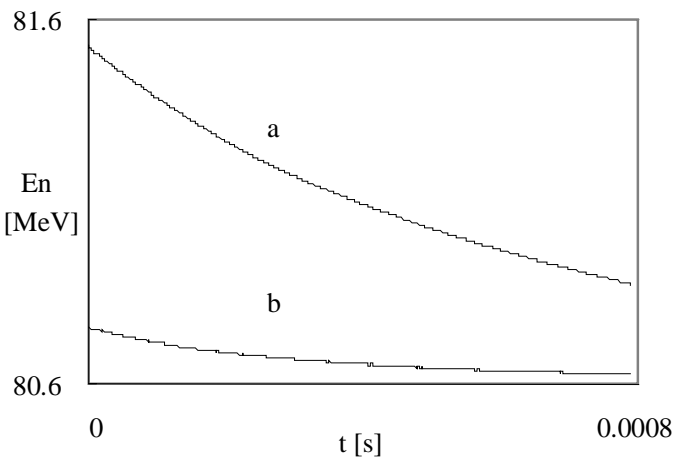

FIG. 13. The computed energy gain when frequencies of the highest $(R / Q)$ modes from the FM passband are set to the nearest harmonics: (a) without compensation, (b) with the injection time compensation.

remedy is to keep the frequencies of these modes between harmonics. This requires an accuracy in the fabrication of irises of better than $\pm 0.2 \mathrm{~mm}$. Further simulations, for the higher $Q$ values, gave almost the same result due to the short beam time, $800 \mu \mathrm{s}$. As an example, the beam induced voltage vs $Q$ for a mode with $(R / Q)=1 \Omega$ and a beam current of $8 \mathrm{~mA}$ is shown in Fig. 14. It can be seen that the voltage rather weakly depends on $Q$ for values higher than $10^{7}$.

\section{FINAL REMARKS}

The proposed superstructure is not yet proven experimentally. In the near future copper models of the superstructure and HOM coupler will be ready. The rf measurements on those models should help us to verify the computation we have done up to now for the superstructure made of four 7-cell cavities. We will be able to check on the copper models tuning and field profile adjustment, transient state in individual cells, HOM damping scheme, coupling to FM coupler, and influence of machining errors.

To check the numerical simulation of the bunch-tobunch energy spread a $\mathrm{Nb}$ prototype must be built and tested with the beam.

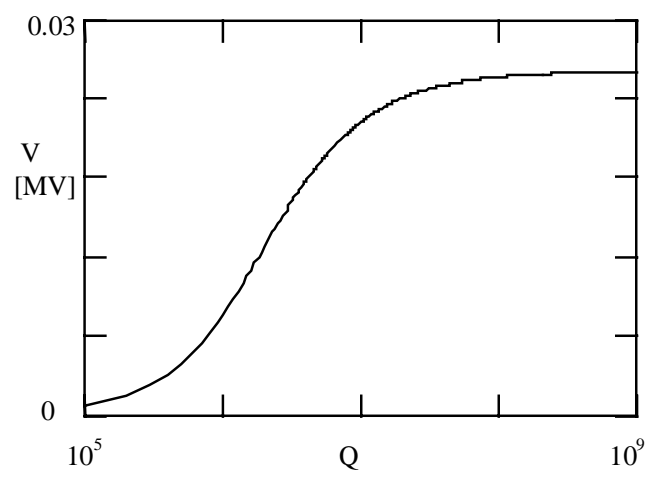

FIG. 14. The induced voltage vs $Q$, for $(R / Q)=1 \Omega$, a beam current of $8 \mathrm{~mA}$, and a beam on time of $800 \mu \mathrm{s}$. 


\section{ACKNOWLEDGMENTS}

We would like to express our gratitude to R. Brinkmann, D. Proch, P. Schmüser, N. Walker, J. Weisend, and the TESLA Collaboration group for many helpful discussions.

[1] D. Edwards, TESLA Report No. 95-01, 1995.

[2] B. Aune and D. Trines, in Proceedings of the Particle Accelerator Conference, Vancouver, Canada, 1997 (IEEE, Piscataway, NJ, 1998).

[3] B. Dwersteg and Q. Yufang, DESY Report No. M-89-08, 1989.

[4] D. Nagel, E. Knapp, and B. Knapp, Rev. Sci. Instrum. 38, 1583 (1967).

[5] G. A. Loew and R. B. Neal, in Linear Accelerators, edited by P.M. Lapostolle and A.L. Septier (North-Holland, Amsterdam, 1969).

[6] J. Sekutowicz, TTF Meeting, IPN internal report, Orsay, France, 1997.
[7] H. Kaiser (private communication).

[8] V. Palmieri et al., in Proceedings of the 7th Workshop on SRF, Gif-sur-Yvette, 1995 (Institut National de Physique Nucléaire at de Physique des Particules, Gif-sur-Yvette, 1996).

[9] R. Brinkmann, in Proceedings of the Particle Accelerator Conference, Dallas, 1995 (IEEE, Piscataway, NJ, 1995), Vol. 1.

[10] J. Sekutowicz, M. Ferrario, and C. Tang, in Proceedings of LC'97, Zvenigorod, 1997 (Institute of Nuclear Physics, Portvino, 1997).

[11] M. Ferrario, A. Mosnier, L. Serafini, F. Tazzioli, and J. M. Tessier, Part. Accel. 52, 1-30 (1996).

[12] R. Klatt et al., in Proceedings of the 1986 Linear Accelerator Conference, Stanford, California (SLAC, Stanford, CA, 1996).

[13] M. Dohlus (private communication).

[14] D. Proch (private communication).

[15] J. Sekutowicz, M. Ferrario, and C. Tang, in Proceedings of the TTF Meeting, DESY, 1998 (DESY, Hamburg, 1998) (TESLA Report No. 98-05). 